\title{
Asian Robotic Gynecology Congress 2019, moving closer to the goal
}

\section{Hyewon Chung}

Department of Obstetrics and Gynecology, Keimyung University Dongsan Medical Center, Daegu, Korea

The Asian Robotic Gynecology Congress 2019 (ARGC 2019) was held at the Sheraton Seoul Palace Gangnam Hotel, Seoul, South Korea, from Thursday, October 24 to Friday, October 25, 2019.

ARGC 2019 was the fifth gathering since the Asian Society of Gynecological Robotic Surgery (ASGRS) held its first international academic conference in 2015, which was a joint event with other surgery departments. ARGC 2019 was the first academic congress on robotic surgery in ob- stetrics and gynecology to be held in Asia.

This conference facilitated active exchange of knowledge among 150 international surgeons and professors from 15 countries (Figs. 1, 2). Given this outreach, this solo international conference in robotic surgery in obstetrics and gynecology was a resounding success.

The number of participants and surgeons from different countries exceeded expectations. The conference hall was crowded from the morning of the first day, and the attend-

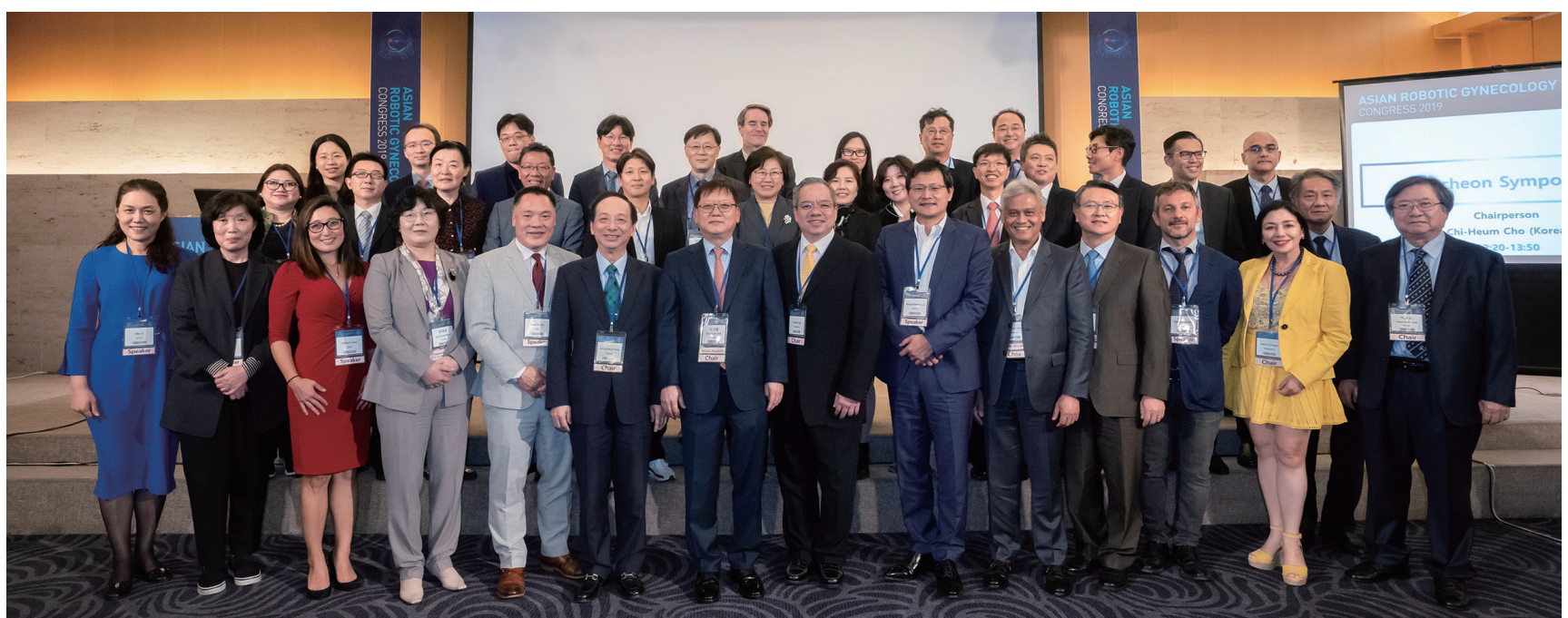

Fig. 1. Invited speakers together before gala dinner.

- Received: August 7, 2020 • Accepted: August 10, 2020

- Correspondening author: Hyewon Chung

Department of Obstetrics and Gynecology, Keimyung University Dongsan Medical Center, 1035 Dalgubeol-daero, Dalseo-gu, Daegu 42601, Korea

E-mail: hyewonny81@naver.com

This is an Open Access article distributed under the terms of the Creative Commons Attribution Non-Commercial License (http://creativecommons.org/ licenses/by-nc/4.0) which permits unrestricted non-commercial use, distribution, and reproduction in any medium, provided the original work is properly cited. 


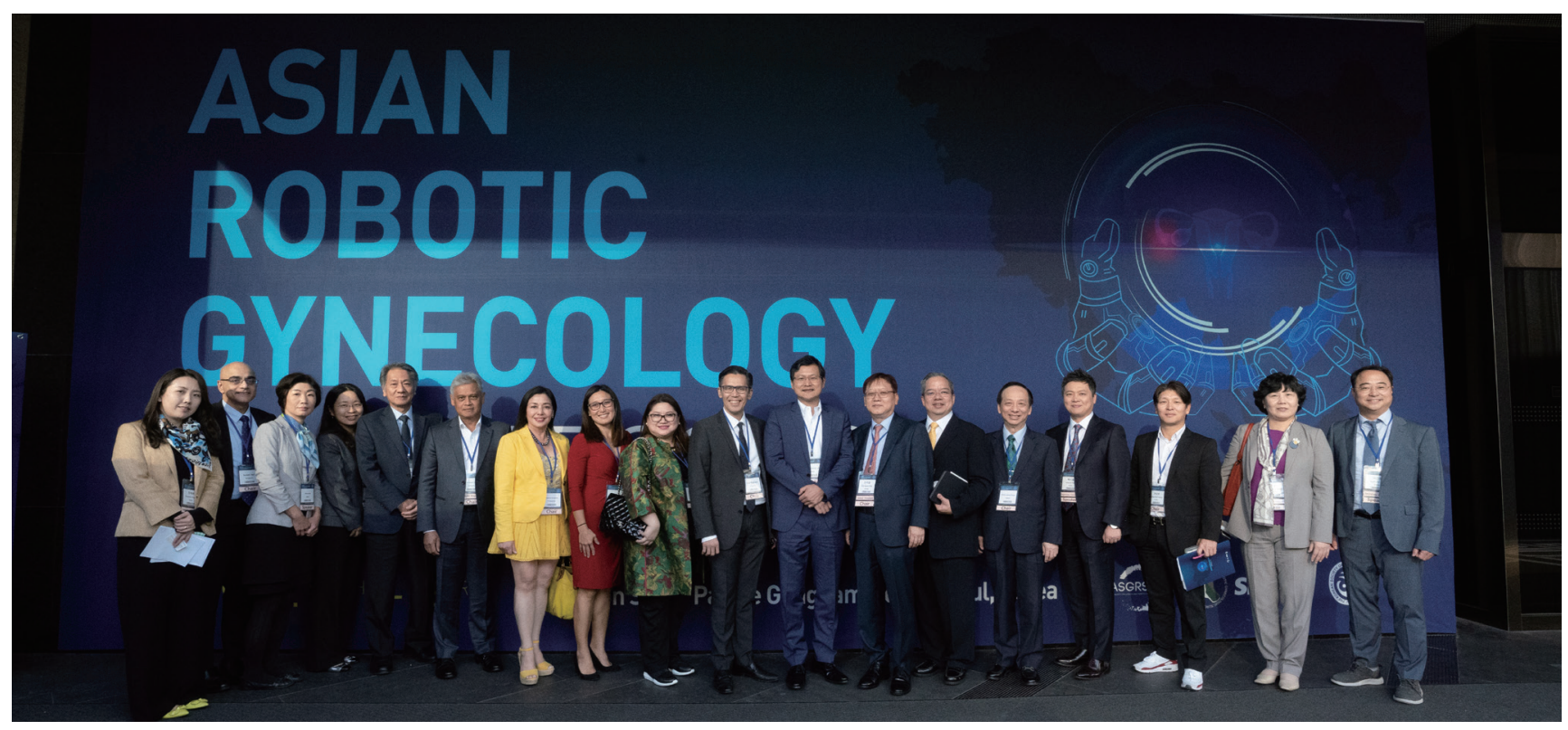

Fig. 2. Invited speakers of special session for nurses.

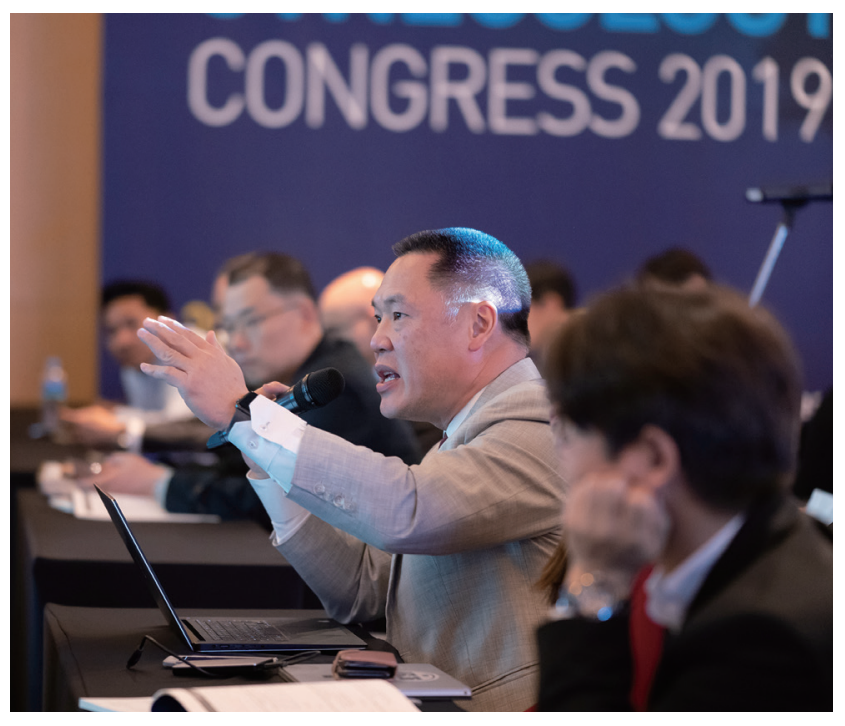

Fig. 3. Peter Lim, Center of Hope, Reno, Nevada, USA.

ees' enthusiasm for robotic surgery pervaded the huge conference hall. The sessions included high-quality lectures by leading surgeons, not only from Korea but all over the world. Given the interest and passion of the various surgeons in robotic surgery, this event was not simply an academic conference, but a place for the communication and exchange of opinions in an enabling atmosphere.

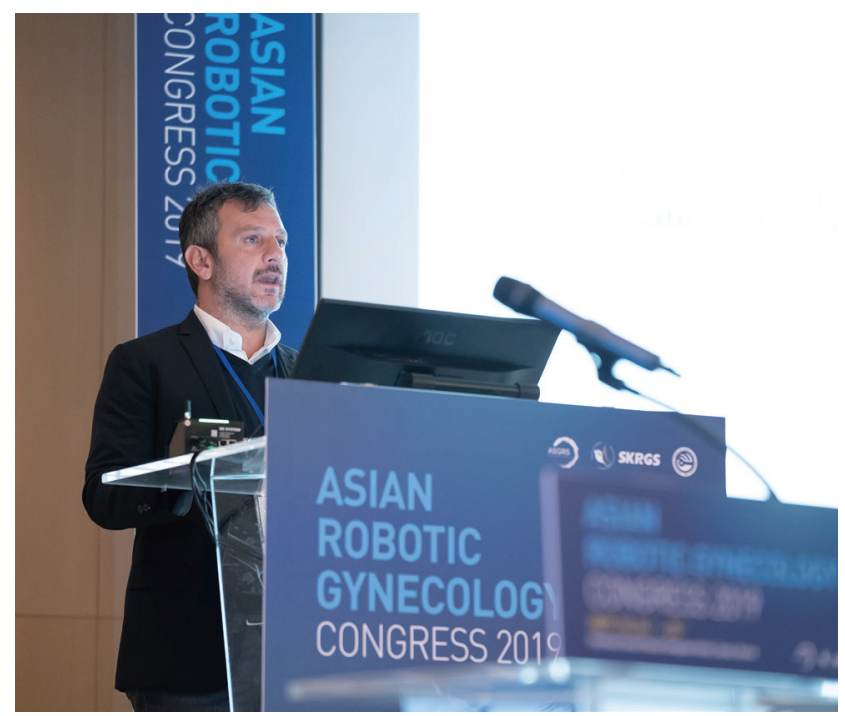

Fig. 4. Valero Gallotta, Fondazione Policlinico Universitario "Agostino Gemelli", IRCCS, Università Cattolica del Sacro Cuore, Rome, Italy.

\section{THURSDAY, OCTOBER 24}

The first key lecture session on Day 1 concerned cervical cancer. The results of the Laparoscopic Approach to Cervical Cancer (LACC) trial published last year showed that various considerations exist with regard to minimally invasive cervical cancer surgery. Peter Lim of Nevada University, USA, gave a lecture on factors such as tumor size and 
spillage that could affect the outcome of robotic radical hysterectomies (Fig. 3). He also stated that much experience is required to evaluate minimally invasive radiological hysterectomies and achieve optimal results, and that the parametrium disconnection method and uterine manipulator appear to be involved in poor outcomes. Henrik Falconer of Karolinska University, Sweden, introduced the results of the Robot-assisted Approach to Cervical Cancer trial, an international multicenter randomized controlled trial that began in May 2019.

The second key lecture session included lectures by Xiaoming Guan of Baylor Medical School, USA, and Valero Gallotta, Italy, on the role of robotic surgery in endometriosis and the application of robotic surgery in the USA (Fig. 4). The discussion covered all aspects of endometriosis in elderly patients and stressed that multi-pronged approaches in robotic surgery can be safely applied.

\section{FRIDAY, OCTOBER 25}

The key lectures on Day 2 included sessions on the role and approach of robotic surgery in treating ovarian cancer and endometrial cancer as well as the future of ASGRS. Ovarian cancer surgery is known to pose some difficulties due to the limitations of the robot platform itself, because it requires extensive control. However, as developments continue to be made in minimally invasive surgery, robotic surgery is bound to play a crucial role in ovarian cancer treatment, and future discussions should focus on this topic.

Peter Lim and Valerio Gallotta shared their experiences, tips, and tricks for robotic surgery in primary and recurrent ovarian cancer. Various opinions were exchanged as to the fact that these techniques could be applied safely on selected patients. Henrik Falconer delivered a lecture on robotic surgery for high-risk endometrial cancer. He introduced ro-

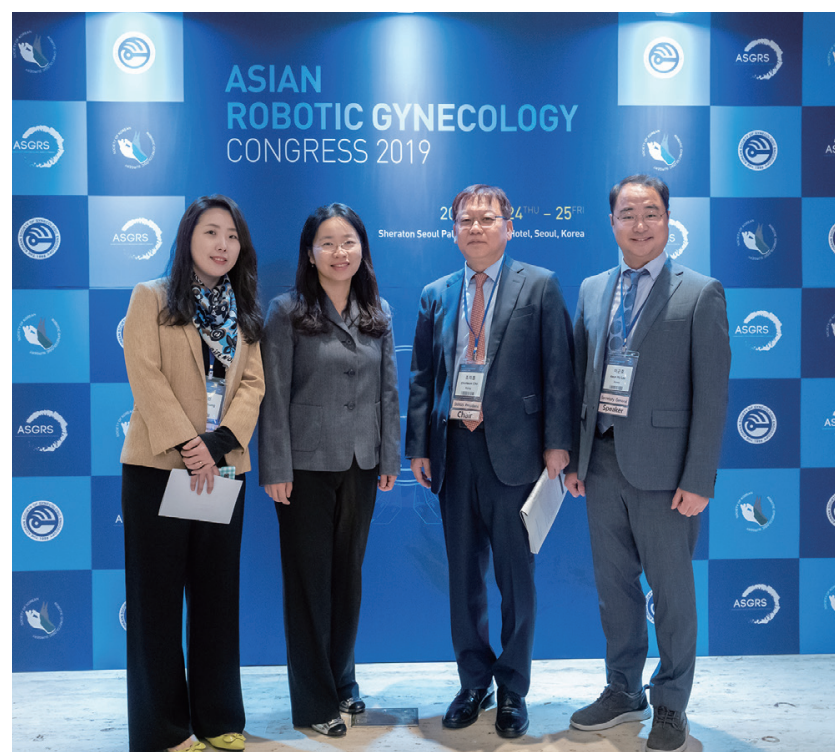

Fig. 5. Asian Robotic Gynecology Congress 2019 Committee.

bot-assisted laparoscopic surgery (RALS), which offers advantages in reducing the conversion rate of open laparotomies and the learning curve. He also lectured on the advantages of applying RALS to high-risk endometrial cancer, and the usefulness of sentinel lymph node biopsies in para-aortic lymph node resection.

In addition, the conference included lectures on the latest insights gained and tricks used by surgeons while performing robotic surgery in various gynecological cases, and active discussions took place freely in a congenial atmosphere of scientific inquiry. We were thus able to move closer to the goal we had envisaged for ARGC 2019. This conference showed me that interest in robot surgery in obstetrics and gynecology was high in Asia as well as Korea. I expect that the future holds some important developments in micro-invasive surgery and robotic surgery. I also felt a sense of pride because Korea's superior status in the field of robotic surgery was evident (Fig. 5). 